\title{
Complete genomic sequence analysis of infectious bronchitis virus Ark DPI strain and its evolution by recombination
}

\author{
Arun Ammayappan ${ }^{1,2}$, Chitra Upadhyay ${ }^{1}$, Jack Gelb Jr ${ }^{3}$ and \\ Vikram N Vakharia*1
}

Address: ${ }^{1}$ Center of Marine Biotechnology, University of Maryland Biotechnology Institute, Baltimore, 701 East Pratt Street, Baltimore, Maryland 21202-3101, USA, 2Department of Veterinary Medicine, University of Maryland, College Park, MD 20742, USA and ${ }^{3}$ Avian Biosciences Center, University of Delaware, 531 South College Avenue, Newark, DE 19716-2150, USA

Email: Arun Ammayappan - ammayapp@umbi.umd.edu; Chitra Upadhyay - upadhyay@umbi.umd.edu; Jack Gelb - jgelb@udel.edu; Vikram N Vakharia* - vakharia@umbi.umd.edu

* Corresponding author

Published: 22 December 2008

Virology Journal 2008, 5:157 doi:10.1186/1743-422X-5-157

This article is available from: http://www.virologyj.com/content/5/I/I57

(c) 2008 Ammayappan et al; licensee BioMed Central Ltd.

This is an Open Access article distributed under the terms of the Creative Commons Attribution License (http://creativecommons.org/licenses/by/2.0), which permits unrestricted use, distribution, and reproduction in any medium, provided the original work is properly cited.

\begin{abstract}
An infectious bronchitis virus Arkansas DPI (Ark DPI) virulent strain was sequenced, analyzed and compared with many different IBV strains and coronaviruses. The genome of Ark DPI consists of 27,620 nucleotides, excluding poly (A) tail, and comprises ten open reading frames. Comparative sequence analysis of Ark DPI with other IBV strains shows striking similarity to the Conn, Gray, JMK, and Ark 99, which were circulating during that time period. Furthermore, comparison of the Ark genome with other coronaviruses demonstrates a close relationship to turkey coronavirus. Among non-structural genes, the 5'untranslated region (UTR), 3C-like proteinase (3CLpro) and the polymerase (RdRp) sequences are 100\% identical to the Gray strain. Among structural genes, $\mathrm{SI}$ has $97 \%$ identity with Ark 99 ; S2 has $100 \%$ identity with JMK and $96 \%$ to Conn; $3 \mathrm{~b} 99 \%$, and $3 \mathrm{C}$ to $\mathrm{N}$ is $100 \%$ identical to Conn strain. Possible recombination sites were found at the intergenic region of spike gene, 3 'end of $\mathrm{SI}$ and 3a gene. Independent recombination events may have occurred in the entire genome of Ark DPI, involving four different IBV strains, suggesting that genomic RNA recombination may occur in any part of the genome at number of sites. Hence, we speculate that the Ark DPI strain originated from the Conn strain, but diverged and evolved independently by point mutations and recombination between field strains.
\end{abstract}

\section{Findings}

Avian infectious bronchitis virus (IBV) is a pathogen of domestic chickens that causes acute, highly contagious respiratory disease [1]. IBV is a member of the Coronaviridae, order Nidovirales [2] and its genome consists of a 27.6 $\mathrm{kb}$ single stranded positive-sense RNA molecule that encodes for four structural proteins; the spike (S) glycoprotein, the small envelope (E) protein, the membrane $(\mathrm{M})$ glycoprotein, and the nucleocapsid $(\mathrm{N})$ protein $[3,4]$.
Six subgenomic mRNAs are transcribed from the IBV genome in virus-infected cells. The mRNA 1 contains two large overlapping open reading frames, encoding two polyproteins $1 \mathrm{a}$ and $1 \mathrm{~b}[5]$, among which $1 \mathrm{~b}$ is produced as $1 \mathrm{ab}$ polyprotein by ribosomal frame-shifting mechanism [6].

Many serotypes have been described for IBV, probably due to the frequent point mutations that occur in RNA 
viruses and also due to recombination events demonstrated for IBV [7-9]. For this reason, the characterization of virus isolates existing in the field is very important. The Ark DPI strain was first isolated from Delmarva Peninsula broiler flock $[10,11]$ and it is currently being used as a vaccine in the USA and Europe. In this study, we characterized the entire genome of virulent Ark DPI strain (embryo passage 11) and compared it with other IBV strains and coronaviruses from all over the world.

The Ark DPI virus was inoculated into 9-day-old SPF chicken eggs and allantoic fluid was collected $72 \mathrm{~h}$ post inoculation. The fluid was clarified by low speed centrifugation and clear supernatant was stored at $-80^{\circ} \mathrm{C}$. Genomic RNA was extracted from virus-infected allantoic fluid with Qiagen RNAeasy kit, following the manufacturer's instructions, and stored at $-80^{\circ} \mathrm{C}$ until further use. Oligonucleotides were designed based on consensus sequence of the following IBV strains: Cal 99 [GenBank:AY514485], Mass 41 [GenBank:AY851295] and BJ [GenBank:AY319651]. Overlapping primers were designed in a manner such that each pair of primer covered approximately two kb of genome. The RT-PCR was carried out as described earlier [12] and the RT-PCR products were cloned into pCR2.1 TOPO TA vector (Invitrogen, CA). Plasmid DNA from various clones was sequenced by dideoxy chain termination method, using an automated DNA sequencer (Applied Biosystems, CA). Three independent clones were sequenced for each amplicon to exclude errors that can occur from RT and PCR reactions. The assembly of contiguous sequences and multiple sequence alignments were performed with the GeneDoc software [13]. The pair-wise nucleotide identity and comparative sequence analyses were conducted using Vector NTI Advance 10 software (Invitrogen, CA) and BLAST search, NCBI. Phylogenetic analyses were conducted using the MEGA4 program [14].

The GenBank accession number for the Ark DPI sequence is EU418976. The complete genomes of following strains are obtained from GenBank: TCoVMG10, NC 010800; Beaudette, NC 001451; M41, AY851295; CK/CH/LSD/ 05I, EU637854; A2, EU526388; LX4, AY338732; SAIBK, DQ288927; The accession numbers of IBV gene sequences which are used in this study are as follows: For replicase gene sequences: (a) 5'UTR; Conn, AY392049; Florida, AY392050; CU-T2, AY561724; Ark 99, AY392051; DE072, AY392054;GA98, AY392053; Gray, AY392056; (b) PLpro: Conn, AY392059; Florida, AY392060; CU-T2, AY561734; Ark 99, AY392061; DE072, AY392064; GA98, AY392063; Gray, AY392066 (c) Mpro: Conn, AY392069; Florida, AY392070; CU-T2, AY561744; Ark 99, AY392071; DE072, AY392074; GA98, AY392073; Gray, AY392076; (d) RdRp: Conn, AY392079; Florida, AY392080; CU-T2, AY561754; Ark99, AY392081;
DE072, AY392084; GA98, AY392083; Gray, AY392086. For Structural genes (a) Complete structural genes: HK, AY761141; Vic, DQ490221; KB8523, M21515; TW2296/ 95, DQ646404 (b) S1: Jilin, AY839144; Gray, L18989; Conn, EU526403; Holte, L18988; UK/2/91， Z83976; Qu16, AF349620; JMK, L14070; H120, M21970; GAV-92, AF094817; DE072, AF274435; IS/1366, EU350550; (c) S2: JMK, AF239982; Jilin, AY839146; Holte, AF334685; DE072, AY024337; Conn, AF094818; Gray, AF394180; H120, AF239982; (d) S: Ark 99, L10384; CU-T2, U04739; (e) Gene 3: Jilin, AY846833; Conn, AY942752; CU-T2, U46036; Ark 99, AY942751; Gray, AF318282 (f) M: Jilin, AY846833; JMK, AF363608; Conn, AY942741; H120, AY028295; Gray, AF363607; (g) Gene 5: Jilin, AY839142; Gray, AF469011; Conn, AF469013; DE072, AF203000; (h) N: Jilin, AY839145; Ind/TN/92/03, EF185916; Conn,

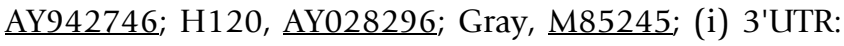
$\mathrm{H} 120, \underline{\mathrm{A} J 278336}$.

The genome of Ark DPI consists of 27,620 nucleotides (nts), excluding poly (A) tail, and comprises ten open reading frames (ORFs) flanked by 5' (528 nts) and 3' (507 nts) untranslated regions (UTRs). The genome organization is ORF1ab (529-20360), ORF2 (20311-23820), ORF3abc [3a, (23820-23993), 3b (23993-24187), 3c (24168-24491)], ORF4 (24469-25140), ORF5ab [5a (25500-25697), 5b (25694-25942)] and ORF6 (2588527114).

The details of genome organization of Ark DPI are shown in Fig. 1. IBV polyprotein is cleaved into 15 cleavage products, among which first two $\mathrm{N}$-terminal products are cleaved by PLpro and rest of the C-terminal products are cleaved by $3 \mathrm{CL}^{\text {pro }}[15]$. The putative domains and their cleavage sites (Fig. 1) are predicted by comparison of amino acid sequences of each non-structural protein (nsp) of Ark DPI with those of IBV-Beaudette which is available in Coronavirus Database (CoVDB) [16]. The nucleotide and the amino acid identity of Ark DPI with other IBVs and coronaviruses are listed in Tables 1, 2, 3. The whole structural gene of Jilin is $100 \%$ identical to Ark DPI, which suggests that Jilin strain is actually Ark DPI, which is currently used as a vaccine in China [17]. The whole genome comparison of IBV strains reveals a close relationship of Ark DPI with Cal 99 (96\% identity), as shown in Fig. 2. Earlier studies have shown that Cal 99 probably evolved from Ark DPI [18].

The complete genome sequence analysis of Ark DPI strain shows striking similarity to the Conn, Gray, JMK, and Ark 99 IBV strains, which were circulating during that time period [1,19-21]. The 5'UTR, PLpro, Mpro and RdRp sequence analysis demonstrates that Ark DPI is $100 \%$ identical to Gray strain, except for PLpro which has $87 \%$ identity, as shown in Table 1. It was suggested that PLpro 


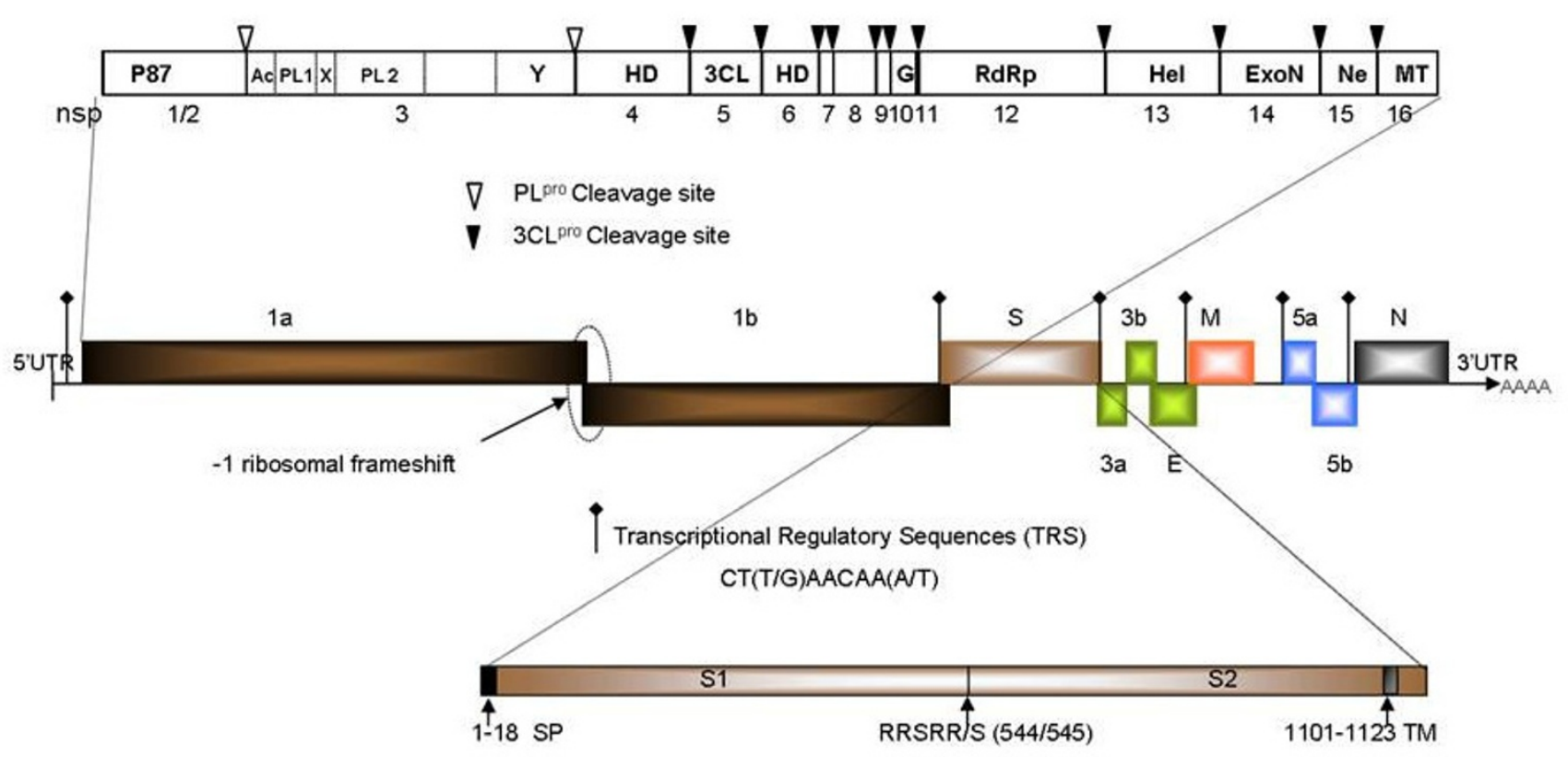

Figure I

Classical Genome Organization of IBV-Ark DPI. The genome of Ark DPI is 27,620 nt long, excluding poly (A) tract. Middle: ten genes and its ORFs. Ribosomal frameshift and position of transcriptional regulatory sequences (TRS) of each gene is indicated. Top: putative domains of ORFla/lb polyprotein: nsp-non-structural protein, Ac-acidic domain, $\mathrm{X}$-unknown domain X, PLI- papain like proteinase I, PL2-papain like proteinase 2; Y-unknown domain Y; HD-hydrophobic domain, 3CL-3C-like proteinase, G-Growth factor like protein, RdRp-RNA dependent RNA polymerase, Hel-helicase, ExoN-exoribonuclease, Nenidoviral uridylate-specific endoribonuclease, MT- 2'-O-ribose methyltransferase. Bottom: details of spike protein; SP-signal peptide, RRSRR/S- spike protein cleavage site between 544 and 545aa, TM-transmembrane domain of spike protein.

gene has high genetic variation because of selection pressure [22]. From this analysis, it appears that genetic mutation may have occurred at PLro gene level. The modern strain GA98 maintains 100\% identity with Ark DPI in replicase proteins and because of unavailability of sequence information for rest of the genome; we speculate that GA98 may be a derivative of the Ark DPI strain.

Analysis of the structural region of Ark DPI clearly demonstrates that it is a chimera of three strains. The S1 gene of Ark DPI is probably derived from Ark 99 (97\% identical) and because of genetic mutations in the S1 region, Ark DPI may have evolved independently. There is an A-T rich sequence TGTGTTGATTATAAT (Fig. 3) at the 3 'terminus of S1 gene ( 300 nts upstream from the end of S1 gene) which is conserved among most of the IBV strains. The S1 gene of Ark 99 maintains its identity with Ark DPI up to this conserved region, but from this point onwards to the end of S2, the nucleotide sequence is $100 \%$ identical to JMK strain. The recombination between JMK and Ark 99 had taken place presumably between above mentioned conserved region and intergenic (IG) region of $S$ gene, which is located 49 nts upstream of start codon of S gene. It is speculated that IG sequences serve as "hot spots" for recombination because of its consensus nature [23]. Gray and JMK strains share 99\% homology both in the S1 and S2 genes of Ark DPI, but JMK shows greater identity than Gray strain, as shown in Table 2. It is interesting that very few residues in the $\mathrm{S} 1$ gene make the Gray strain nephrotropic, whereas JMK is pneumotropic [24].

Out of 174 nts of gene 3a of Ark DPI, last 74 nts are $100 \%$ identical to Conn, whereas first 100 nts are only $86 \%$ identical. Even though it is not clear whether the 5 '-end of 3a was derived from Conn or JMK, but it is evident that the recombination event may have occurred between JMK and Conn at gene $3 \mathrm{a}$. The $3 \mathrm{~b}$ gene of Ark DPI and Conn differed only by two nucleotides and both share 99\% identity, suggesting that $3 \mathrm{~b}$ belongs to Conn strain. From gene $3 \mathrm{c}$ to $\mathrm{N}$ gene, Ark DPI shares $100 \%$ identity with Conn. It is obvious that the entire structural genome, except spike, belongs to Conn strain. Cross protection studies carried out by Gelb and coworkers [11] demonstrated that the birds immunized with Ark DPI showed 
Table I: Percent (\%) nucleotide identity of Ark DPI non-structural genes and ORF Iab, ORF2-6 and complete genome with other IBV strains $\mathbf{a}, \mathrm{b}, \mathrm{c}$

\begin{tabular}{|c|c|c|c|c|c|c|c|}
\hline IBV Strains & 5'UTR & PLpro & Mpro & $R d R p$ & ORFI & ORF2-6 & Complete genome \\
\hline Ark99 & 98 & 87 & 99 & 99 & NA & 96 & NA \\
\hline $\mathrm{A} 2$ & 95 & 83 & 85 & 99 & 86 & 85 & 86 \\
\hline Beaudette & 96 & 85 & 93 & 93 & 91 & 91 & 91 \\
\hline BJ & 96 & 83 & 88 & 93 & 86 & 85 & 86 \\
\hline Cal99* & 100 & 99 & 97 & 99 & 96 & 94 & 96 \\
\hline $\mathrm{CK} / \mathrm{CH} / \mathrm{LSD} / 05 \mathrm{I}$ & 97 & 84 & 88 & 90 & 89 & 90 & 89 \\
\hline Connecticut & 97 & 87 & 100 & 99 & NA & 96 & NA \\
\hline CU-T2* & 100 & 94 & 99 & 99 & NA & 94 & NA \\
\hline DE072 & 100 & 100 & 90 & 90 & NA & NA & NA \\
\hline Florida & 97 & 87 & 100 & 99 & NA & NA & NA \\
\hline GA98* & 100 & 100 & 100 & 100 & NA & NA & NA \\
\hline Gray & 100 & 87 & 100 & 100 & NA & NA & NA \\
\hline Jilin & NA & NA & NA & NA & NA & 100 & NA \\
\hline LX4 & 94 & 83 & 85 & 89 & 87 & 84 & 86 \\
\hline M4I & 97 & 87 & 91 & 94 & 91 & 91 & 91 \\
\hline SAIBK & 92 & 85 & 90 & 90 & 90 & 86 & 89 \\
\hline
\end{tabular}

a Sequences with $>95 \%$ identity are in bold letters

b NA-not analyzed

c Parental strains of Ark DPI are shown in bold letters and immediate derivative of Ark DPI is indicated by asterisk (*).

$95 \%, 90 \%$ and 63\% protection against Conn, Ark 99 and JMK strains, respectively. Indeed, these results suggest that major part of Ark DPI genome was derived from Conn. The level of protection for JMK is $80 \%$, when Ark 99 was used as immunogen [25]. On the other hand, Conn and JMK immunization induces inadequate immunity against Ark-type IBV challenge, suggesting that Ark cross-immunity to JMK and Conn is a one-way relationship $[10,11,26]$.

Recombination hot spots have been demonstrated for IBV isolates by many researchers. These hot spots have been detected in the IG region [23], S1 gene [27], 3' terminus of S2, N and between N gene and 3'UTR $[8,28]$. Some earlier sequencing studies had provided circumstantial evidence of recombination events in field isolates of IBV $[7,29,30]$. More or less recombination sites were detected over the entire genome of coronavirus [31]. Based on these results, we speculate that the Ark DPI strain originated from the Conn strain, but diverged and evolved independently by point mutations and recombination between field strains. These findings suggest that there is high level of genetic diversity among currently circulating IBV serotypes. Most of them come from genetic changes which already exist in the IBV field strains and from IBV live vaccines. So frequent monitoring is highly essential to track the emergence of new variants and is mandatory to develop efficient vaccination strategies to control and prevent IB. 
Table 2: Percent (\%) nucleotide identity of Ark DPI structural genes with other IBV strains a, b, c, d

\begin{tabular}{|c|c|c|c|c|c|c|c|c|c|c|}
\hline BV Strains & SI & S2 & $3 a$ & $3 b$ & $3 c$ & $M$ & $5 a$ & $5 b$ & $N$ & 3'UTR \\
\hline Ark99 & 97(96) & $96(95)$ & 92 & 99 & $96(92)$ & 97(97) & 93 & 97 & $98(98)$ & 97 \\
\hline Beaudette & $81(79)$ & $95(94)$ & 91 & 84 & $88(83)$ & $91(93)$ & 85 & 93 & $93(95)$ & 97 \\
\hline BJ & $77(75)$ & $85(89)$ & 88 & 76 & $87(79)$ & $90(93)$ & NA & NA & $89(93)$ & 87 \\
\hline Cal99* & $87(84)$ & $94(93)$ & 92 & 99 & $94(90)$ & $97(96)$ & 100 & 98 & $95(98)$ & 89 \\
\hline $\mathrm{CK} / \mathrm{CH} / \mathrm{LSD} / 05 \mathrm{I}$ & $78(75)$ & $88(91)$ & 89 & 84 & $(87)$ & $96(96)$ & 99 & 100 & $100(99)$ & 90 \\
\hline Conn & $81(77)$ & $96(96)$ & 92 & 99 & $100(100)$ & $100(100)$ & 100 & 100 & $100(100)$ & NA \\
\hline CU-T2* & $96(94)$ & $93(93)$ & 88 & 98 & $92(87)$ & $88(87)$ & 97 & 99 & $95(96)$ & 97 \\
\hline DE072 & $62(50)$ & $75(76)$ & NA & NA & NA & NA & 98 & 98 & NA & 98 \\
\hline Gray & $83(80)$ & 99(99) & NA & NA & $96(92)$ & 98(98) & 97 & 99 & 97(97) & 98 \\
\hline GAV-92 & $94(92)$ & NA & NA & NA & NA & NA & NA & NA & NA & NA \\
\hline $\mathrm{HI} 20$ & $81(78)$ & NA & NA & NA & NA & $92(94)$ & NA & NA & $93(96)$ & 83 \\
\hline $\mathrm{HK}^{*}$ & $81(78)$ & $96(96)$ & 99 & 100 & $100(100)$ & $100(100)$ & 100 & 100 & $100(100)$ & NA \\
\hline Holte & $83(80)$ & $95(95)$ & NA & NA & NA & NA & NA & NA & NA & NA \\
\hline Ind/TN/92/03 & NA & NA & NA & NA & NA & NA & NA & NA & $92(94)$ & NA \\
\hline IS/I 366 & $78(75)$ & NA & NA & NA & NA & NA & NA & NA & $92(95)$ & NA \\
\hline Jilin* & $100(99)$ & $100(100)$ & 100 & 100 & $100(100)$ & $100(100)$ & 100 & 100 & $100(100)$ & 100 \\
\hline JMK & $84(82)$ & $100(100)$ & NA & NA & NA & $97(98)$ & NA & NA & NA & NA \\
\hline KB8523 & $81(78)$ & $91(92)$ & NA & NA & NA & $93(95)$ & NA & NA & $95(96)$ & NA \\
\hline LX4 & $77(76)$ & $85(88)$ & 86 & 76 & $88(80)$ & $91(91)$ & 82 & 90 & $89(93)$ & NA \\
\hline M4I & $81(78)$ & $95(94)$ & 91 & 85 & $88(83)$ & $91(95)$ & 90 & 97 & $94(95)$ & 97 \\
\hline Qul6 & $84(81)$ & NA & NA & NA & NA & NA & NA & NA & NA & NA \\
\hline SAIBK & $79(77)$ & $87(91)$ & 86 & 83 & $85(80)$ & $89(93)$ & 84 & 96 & $87(92)$ & 88 \\
\hline TW2296/95 & $79(77)$ & $86(90)$ & 86 & 85 & (83) & $91(92)$ & 82 & 96 & $89(92)$ & NA \\
\hline UK/2/9I & $78(76)$ & NA & NA & NA & NA & NA & NA & NA & NA & NA \\
\hline Vic & $81(79)$ & $89(92)$ & 88 & 88 & $88(88)$ & $89(95)$ & 87 & 94 & $90(94)$ & NA \\
\hline
\end{tabular}

a Sequences with $>95 \%$ identity are indicated in bold letters

${ }^{b}$ Amino acid sequences within the parenthesis

c NA-Not Analyzed

${ }^{d}$ Parental strains of Ark DPI are shown in bold letters and immediate derivative of Ark DPI is indicated by asterisk (*). 
Table 3: Percent (\%) amino acid identity of Ark DPI replicase and structural proteins to other coronaviruses c, d

\begin{tabular}{|c|c|c|c|c|c|c|c|}
\hline Coronaviruses $^{\mathrm{a}}$ & $3 C L^{p r o}$ & RdRp & $S$ & $E$ & $M$ & $\mathrm{~N}$ & Complete genome ${ }^{b}$ \\
\hline BatCoV & 40 & 70 & 22 & 11 & 29 & 25 & 46 \\
\hline $\mathrm{BCoV}$ & 41 & 67 & 21 & 13 & 30 & 24 & 47 \\
\hline ECoV & 41 & 67 & 22 & 13 & 30 & 25 & 47 \\
\hline FCoV & 45 & 62 & 22 & 16 & 23 & 23 & 48 \\
\hline HCoV 229E & 40 & 63 & 23 & 12 & 26 & 23 & 49 \\
\hline TGEV & 46 & 61 & 22 & 20 & 25 & 26 & 49 \\
\hline MHV A59 & 40 & 69 & 21 & 14 & 31 & 26 & 46 \\
\hline SARS CoV & 46 & 68 & 21 & 17 & 29 & 22 & 45 \\
\hline SWI & 56 & 79 & 25 & 28 & 36 & 35 & 50 \\
\hline TCoV & 96 & 98 & 34 & 91 & 97 & 95 & 88 \\
\hline
\end{tabular}

a BatCoV, Bat coronavirus; FCoV, feline coronavirus; HCoV, human coronavirus; BCoV, bovine coronavirus; MHV, mouse hepatitis virus; SARS-

$\mathrm{CoV}$, severe acute respiratory syndrome coronavirus; SWI, beluga whale coronavirus; TCoV, turkey coronavirus.

b Percent nucleotide identity of entire genome

c Sequences with $>50 \%$ identity are in bold letters

d Gene in bold letters (RdRP) is highly conserved; TCoV exhibits significant identity with IBV-Ark DPI (marked in bold letters).

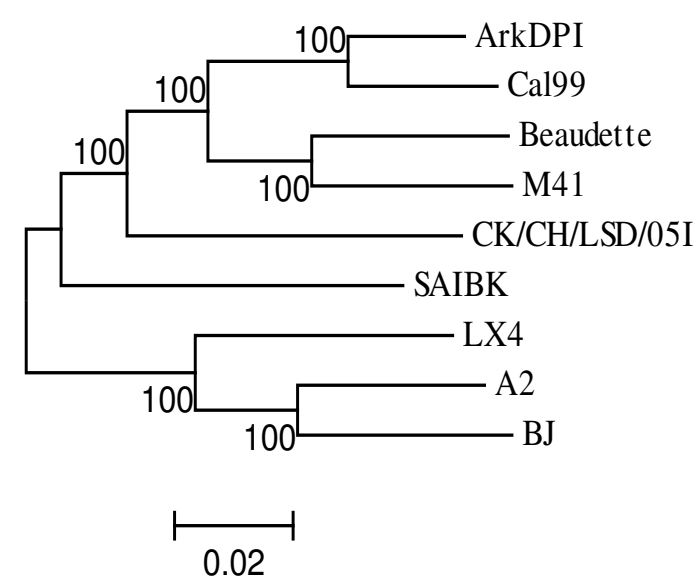

Figure 2

Phylogenetic tree analysis of complete Ark DPI genome sequence with other IBV strains. Phylogenetic tree analysis was conducted by neighbor-joining method using bootstrap analysis ( 100 replications). The scale at the bottom indicates the number of substitution events.

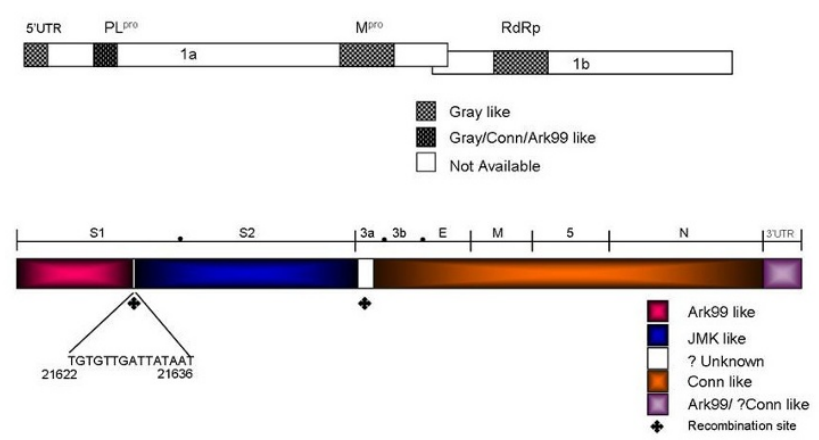

Figure 3

Schematic presentation of the structural region of Ark DPI genome. Entire genome of Ark DPI was analyzed for its similarity with other IBV strains. Top panel: 5'UTR \& ORFI. Shadowed regions were used for comparative analysis. 5'UTR-5'-untranslated region; PLI-papain like proteinase I; Mpro-main or 3C-like proteinase; RdRp-RNAdependent-RNA polymerase. Bottom panel: ORF2 to 3'UTR. Structural genes and their ORFs are marked by $(\mathbf{O})$. Conserved sequence TGTGTTGATTATAAT in SI gene is shown; denotes plausible recombination site in Ark DPI. 


\section{Competing interests}

The authors declare that they have no competing interests.

\section{Authors' contributions}

VNV and JG conceived the study. AA planned the experimental design, AA and CU carried out cloning and sequencing. AA drafted the manuscript. All authors critically reviewed and approved the final manuscript.

\section{Acknowledgements}

The project was supported by the National Research Initiative of the USDA Cooperative State Research, Education and Extension Service; grant number 2004-35204-I48I4 to V.N.V. and J.G.

\section{References}

I. Chomiak TW, Luginbuhl RE, Jungherr EL: The propagation and cytopathogenic effect of an egg-adapted strain of infectious bronchitis virus in tissue culture. Avian Dis 1958, 2:456-465.

2. Cavanagh D, Naqi S: Infectious Bronchitis. In Diseases of Poultry IIth edition. Edited by: Saif YM, Barnes HJ, Glisson JR, Fadly AM McDougald LR, Swayne DE. Ames: lowa State University Press; 2003:101-120.

3. Spaan W, Cavanagh D, Horzinek MC: Coronaviruses: structure and genome expression. J Gen Virol 1988, 69:2939-2952.

4. Sutou S, Sato S, Okabe T, Nakai M, Sasaki N: Cloning and sequencing of genes encoding structural proteins of avian infectious bronchitis virus. Virology 1988, 165:589-595.

5. Boursnell MEG, Brown TDK, Foulds IJ, Green PF, Tomely FM, Binns $M M$ : Completion of the sequence of the genome of the coronavirus avian infectious bronchitis virus. J Gen Virol 1987, 68:57-77.

6. Brierley I, Digard P, Inglis SC: Characterization of an efficient coronavirus ribosomal frameshifting signal: Requirement for an RNA pseudoknot. Cell 1989, 57:537-547.

7. Cavanagh D, Davis PJ, Cook J: Infectious bronchitis virus: evidence for recombination within the Massachusetts serotype. Avian Path 1992, 21:401-408.

8. Kottier SA, Cavanagh D, Britton P: Experimental evidence of recombination in coronavirus infectious bronchitis virus. Virology 1995, 21 3:569-580.

9. Gelb J, Weisman Y, Ladman BS, Meir R: SI gene characteristics and efficacy of vaccination against infectious bronchitis virus field isolates from the United States and Israel (1996 to 2000). Avian Pathol 2005, 34:194-203.

10. Gelb ], Perkins BE, Rosenberger IK, Allen PH: Serologic and crossprotection studies with several infectious bronchitis virus isolates from Delmarva-reared broiler chickens. Avian Dis 1981, 25:655-666.

II. Gelb J, Leary JH, Rosenberger JK: Prevalence of Arkansas-type infectious bronchitis virus in Delmarva Peninsula chickens. Avian Dis 1983, 27:667-678.

12. Brandt M, Yao K, Liu M, Heckert RA, Vakharia VN: Molecular determinants of virulence, cell tropism, and pathogenic phenotype of infectious bursal disease virus. I Virol 2001, 75: I I974-I1982.

13. Nicholas KB, Nicholas HBJ, Deerfield DW: GeneDoc: analysis and visualization of genetic variation. EMBNEW NEWS 1997, 4: I4.

14. Tamura K, Dudley J, Nei M, Kumar S: MEGA4: molecular evolutionary genetics analysis (MEGA) software version 4.0. Mol Biol Evol 2007, 24: I596-I599.

15. Ziebuhr J, Snijder EJ, Gorbalenya AE: Virus-encoded proteinases and proteolytic processing in the Nidovirales. J Gen Virol 2000 81:853-879.

16. Huang H, Lau SKP, Woo PCY, Yuen K: CoVDB: a comprehensive database for comparative analysis of coronavirus genes and genomes. Nucleic Acids Res 2007, 36:1-8.

17. Liu S, Chen J, Han Z, Zhang Q, Shao Y, Kong X, Tong G: Infectious bronchitis virus: $S I$ gene characteristics of vaccines used in China and efficacy of vaccination against heterologous strains from China. Avian Pathol 2006, 35:394-399.
18. Mondal SP, Cardona Cl: Genotypic and phenotypic characterization of the California 99 (Cal99) variant of infectious bronchitis virus. Virus Genes 2007, 34:327-34I.

19. Winterfield RW, Hitchner SB, Appleton GS: Immunological characteristics of a variant of infectious bronchitis virus isolated from chickens. Avian Dis 1964, 8:40-47.

20. Winterfield RW, Hitchner SB: Etiology of an infectious nephritisnephrosis syndrome of chickens. Am J Vet Res 1962, 23: $1273-1279$.

21. Johnson RB, Marquardt WW, Newman JA: A new serotype of infectious bronchitis virus responsible for respiratory disease in Arkansas broiler flocks. Avian Dis 1973, 17:518-523.

22. Mondal SP, Cardona CJ: Comparison of four regions in the replicase gene of heterologous infectious bronchitis virus strains. Virology 2004, 324:238-248.

23. Lee CW, Jackwood MW: Evidence of genetic diversity generated by recombination among avian coronavirus IBV. Arch Virol 2000, | 45:2135-2|48.

24. Kwon HM, Jackwood MW: Molecular cloning and sequence comparison of the SI glycoprotein of the Gray and JMK strains of avian infectious bronchitis virus. Virus Genes 1995, 9:219-229.

25. Hofstad MS: Cross-immunity in chickens using seven isolates of avian infectious bronchitis virus. Avian Dis 1981, 25:650-654.

26. Johnson RB, Marquardt WW: The neutralizing characteristics of strains of infectious bronchitis virus as measured by the constant-virus variable- serum method in chicken tracheal cultures. Avian Dis 1975, 19:82-90.

27. Callison S, Hilt D, Jackwood M: Using DNA shuffling to create novel infectious bronchitis virus SI genes: Implications for S I gene recombination. Virus Genes 2005, 3 I:5-I I.

28. Jia W, Karaca K, Parrish DR, Naqi SA: A novel variant of avian infectious bronchitis virus resulting from recombination among three different strains. Arch Virol 1995, 140:259-27I.

29. Cavanagh D, Davis PJ: Evolution of avian coronavirus IBV: Sequence of the matrix glycoprotein gene and intergenic region of several serotypes. J Gen Virol 1988, 69:621-629.

30. Wang L, Junker D, Collisson EW: Evidence of natural recombination within the SI gene of infectious bronchitis virus. Virology 1993, 192:710-716.

31. Lai MMC: RNA recombination in animal and plant viruses. Microbiol Rev 2000, 56(I):6I-79.

Publish with Biomed Central and every scientist can read your work free of charge

"BioMed Central will be the most significant development for disseminating the results of biomedical research in our lifetime. "

Sir Paul Nurse, Cancer Research UK

Your research papers will be:

- available free of charge to the entire biomedical community

- peer reviewed and published immediately upon acceptance

- cited in PubMed and archived on PubMed Central

- yours - you keep the copyright 